\title{
COMMENT
}

Check for updates

\section{Simultaneous development of zanubrutinib in the USA and China}

Guanqiao Li(i) ${ }^{1}$, Xiaozhen Liu ${ }^{1}$ and Xiaoyuan Chen (D) $^{1,2 凶}$

Zanubrutinib was recently granted expedited approval by the USA and Chinese drug regulatory authorities for the treatment of mantle cell lymphoma, thus becoming the first investigational new drug discovered in China to achieve simultaneous development in both countries. Here, we provide an overview of the regulatory processes and considerations of the two health authorities and discuss the pathways of concurrent review and approval.

Simultaneous drug development in multiple countries can accelerate regulatory approvals and provide broader accessibility. Ground-breaking reforms in the Chinese regulatory environment have supported the incorporation of China into sponsors' synchronized drug development plans ${ }^{1}$. Meanwhile, the Chinese biopharma sector has started to develop novel therapeutics for the international market ${ }^{2}$. Indeed, the BTK inhibitor zanubrutinib recently became the first new chemical entity discovered in China to receive initial international approval by the FDA in the USA, as well as approval by the Chinese National Medical Products Administration (NMPA), based primarily on data from a pivotal study conducted in China ${ }^{3,4}$. Considering these similar expedited approvals, we compare the data requirements, review considerations and processes of the two regulatory agencies and delineate potential pathways for simultaneous review and approval of medicines in China and other countries.

Zanubrutinib is a second-generation BTK inhibitor (Supplementary Table 1) that was discovered by the Chinese company BeiGene in 2012. A first-in-human study of this agent in patients with B cell malignancies began in Australia in 2014, with expansion cohorts opened in the USA and other countries in 2015 (BGB3111-AU-003; Supplementary Table 2). On the basis of preliminary efficacy data, an agreement was reached in December 2016 between the NMPA Centre for Drug Evaluation (CDE) and the sponsor on potential Conditional Approval based primarily on one single-arm phase II trial (BGB-3111-206) involving patients with relapsed and/or refractory mantle cell lymphoma (R/R MCL) in China. The NMPA new drug application (NDA) including data from this pivotal trial was submitted in August 2018, resulting in Conditional Approval on 3 June 2020 (Supplementary Fig. 1a) ${ }^{3}$.

Similarly, the FDA agreed to data from BGB-3111-206 and BGB-3111-AU-003 being used to support approval during a pre-NDA meeting held in August 2018, but required longer follow-up monitoring to document the durability of responses ${ }^{4}$. The US NDA was filed in June 2019, 10 months later than the Chinese NDA. Subsequently, the FDA granted Accelerated Approval in the setting of R/R MCL on 14 November 2019 (Supplementary Fig. 1a) ${ }^{4}$.

Despite sequential NDA submissions to the NMPA and the FDA, the final NDA packages at the time of approval included similar core datasets ${ }^{4,5}$. The primary efficacy-evaluable population comprised 86 patients in the pivotal BGB-3111-206 study and another 32 patients with R/R MCL in BGB-3111-AU-003 (Supplementary Table 2). The safety analysis was also supported by data from 511 patients with other B cell malignancies included in studies BGB-3111-1002, BGB-3111-205 and BGB-3111-210 (REF.). Rolling submissions further enabled the safety data to be updated during regulatory review by the NMPA ${ }^{5}$.

Zanubrutinib induced frequent and durable responses in BGB-3111-206 and BGB-3111-003 (Supplementary Table 2) ${ }^{4}$. Notably, the complete response rate in patients with R/R MCL (59\% in BGB3111-206) seemed higher than observed with the approved BTK inhibitors, ibrutinib or acalabrutinib (Supplementary Table 3). Overall, the compelling efficacy data, together with evidence of good tolerability in a large safety population, convinced both agencies to approve zanubrutinib through expedited pathways ${ }^{4,5}$.

To our knowledge, this was the first FDA approval based on pivotal data mainly from patients in China. On average, only $22 \%$ of patients included in previous pivotal trials of anticancer drugs approved by the FDA were of Asian ethnicity ${ }^{6}$. Key considerations by the FDA when accepting the predominantly Chinese data included population representativeness, ethnic differences and sufficient safety data from white patients ${ }^{4}$. The NMPA CDE, likewise, has an emphasis on population generalizability, data extrapolation and consistency between studies when deciding to accept foreign data. As a condition of expedited approval, both agencies mandated the efficacy of zanubrutinib be validated in a 
post-approval confirmatory phase III trial (BGB-3111306; NCT04002297) $)^{4,5}$.

The two expedited approvals after priority reviews of comparable datasets underscore the scientific tenets of drug regulation and concordant regulatory requirements; however, the 6-month lag in the NMPA approval suggests discrepant mechanisms of drug review and approval. Procedurally, both agencies conduct a pre-NDA meeting, NDA acceptance, filing review, technical review and on-site inspections for Good Clinical or Manufacturing Practice (GCP or GMP) before official approval. Nevertheless, discrepancies in the review cycles and sequence of procedures widened the time gap between the two approvals. For example, the FDA has stringent NDA acceptance criteria to promote a single-cycle review. By contrast, one or two rounds of data supplements and review are generally required by the NMPA, as exemplified in the approval of zanubrutinib (Supplementary Fig. 1b). Moreover, technical review and on-site inspection are conducted in parallel by the FDA, whereas inspections by the NMPA are generally triggered after review and verification of the specifications and processes of drug manufacturing. In the case of zanubrutinib, GCP inspection occurred after completion of the primary review, with GMP inspection after supplemental review (Supplementary Fig. 1b). Taking a risk-based standpoint, the NMPA conducted a full-scale inspection of the manufacturing facility in China because it is a brand-new plant. The FDA instead implemented spot-check inspection of the contract manufacturer in the USA, given their record of good compliance with the required standards. Lastly, requirements of testing of drug samples collected during on-site inspections in China, and not in the USA, further extended the timeline.

The 21-month NDA approval for zanubrutinib in China is longer than the average of 12-14 months required for priority review of other drugs in 2019. Several domestic new drugs achieved approval in China within 12 months of NDA, including two anti-PD-1 antibodies (toripalimab and sintilimab) and the third-generation EGFR inhibitor almonertinib. In retrospect, some external factors might also have contributed to the longer time required for approval of zanubrutinib, such as the COVID-19 pandemic of 2020 (which might have delayed site inspections).

Despite remarkable improvements in drug review in China, several future priorities should be considered, including optimized review processes, detailed requirements and sufficient resourcing. Notably, initiatives to optimize processes are already in place. According to the revised Chinese Drug Registration Regulations (DRR) effective from 1 July 2020, technical review will be conducted in parallel with site inspections, with a stipulated time limit of 130 working days. The DRR also encourages reduction of the number of review cycles, which might prompt the enactment of additional new policies to refine data requirements and standardize processes. Furthermore, real-time review prior to formal NDA submission might further shorten review durations. For example, since 2018, the FDA has implemented rolling data review via the Real-Time Oncology Review programme, in order to expedite the review process while managing workloads. A lack of sufficient resources has always been a key impediment to efficiency at the NMPA and even the $\mathrm{FDA}^{2,7}$. In this regard, building expertise to strengthen science-based and professional judgements is of equal importance to simply recruiting additional staff. For this reason, the NMPA is now collaborating with academia to provide comprehensive training programmes and undertake regulatory research.

Frequent exchange of information and opinions between authorities would further enable the realization of concurrent drug review and approval in multiple countries. Since 2019, the FDA has been collaborating with regulatory agencies in Australia and Canada to conduct drug reviews concurrently under the Project Orbis programme ${ }^{8}$. In today's innovative $\mathrm{R} \& \mathrm{D}$ ecosystem and improving regulatory environment, China is ready to join the dialogue with other top agencies. In the near future, joint review between regulatory authorities including the NMPA is anticipated to realize simultaneous drug development and approval.

1. Wang, M. Clinical trials and drug approvals continue to accelerate in China. Lancet Oncol. 18, 855 (2017).

2. Zhou, O., Chen, X. Y. Yang, Z. M. \& Wu, Y. L. The changing landscape of clinical trial and approval processes in China. Nat. Rev. Clin. Oncol. 14, 577-583 (2017).

3. National Medical Products Administration. National Medical Products Administration Approves Zanubrutinib http:// www.nmpa.gov.cn/WS04/CL2056/377969.html (2020).

4. U.S. Food and Drug Administration. Drug Approval Package: BRUKINSA https://www.accessdata.fda.gov/drugsatfda_docs/ nda/2019/2132170rig 1 s000TOC.cfm (2019).

5. Nasdaq Investors. BeiGene Announces the Approval of BRUKINSA ${ }^{\text {tw }}$ (Zanubrutinib) in China for Patients with Relapsed/Refractory Chronic Lymphocytic Leukemia or Small Lymphocytic Lymphoma and Relapsed/Refractory Mantle Cell Lymphoma http://ir.beigene. $\mathrm{com} /$ news-releases/news-release-details/beigene-announcesapproval-brukinsatm-zanubrutinib-china (2020).

6. Ramamoorthy, A. et al. Demographic composition of select oncologic new molecular entities approved by the FDA between 2008 and 2017. Clin. Pharmacol. Ther. 104, 940-948 (2018).

7. The New York Times. The F.D.A. Is in Trouble. Here's How to Fix It https://www.nytimes.com/2020/01/11/opinion/sunday/ fda-commissioner-stephen-hahn.html (2020).

8. Morrison, C. Fresh from the biotech pipeline-2019. Nat. Biotechnol. 38, 126-131 (2020)

\section{Acknowledgements}

We gratefully acknowledge extensive contributions of Yi-long Wu (Guangdong General Hospital), Ling Su (Shenyang Pharmaceutical University), Yangzhong Zhou (Peking Union Medical College Hospital), Ning Zhang and Lianjie Ren (Chinese Center for Drug Evaluation (CDE)) and Zili Li (Janssen Research \& Development) for discussions relating to the composition and content of this manuscript. We are also grateful to Pharmcube (Beijing) for providing the public data on zanubrutinib.

\section{Competing interests}

The authors declare no competing interests.

Supplementary information

Supplementary information is available for this paper at https://doi. org/10.1038/s41571-020-0414-y.

\section{RELATED LINKS}

Chinese Drug Registration Regulations: http://www.nmpa.gov.cn/WS04/ CL2077/376150.html

Project Orbis: https://www.fda.gov/about-fda/oncology-center-excellence/ project-orbis

Real-Time Oncology Review: https://www.fda.gov/about-fda/oncology-cente excellence/real-time-oncology-review-pilot-program 